\title{
Efecto Terapéutico de los Exosomas en el ACV Isquémico en Animales de Experimentación
}

\author{
Therapeutic Effect of Exosomes on Ischemic Stroke in Experimental Animals
}

\author{
Carvallo, $\mathbf{P}^{*, * * *}$ \& Astudillo, $\mathbf{P}^{* * * * * * * *, * * * * * *}$
}

CARVAllo, P. \& ASTUDILlO, P. Efecto terapéutico de los exosomas en el ACV isquémico en animales de experimentación. Int. J. Morphol., 34(4):1300-1307, 2016.

RESUMEN: La posibilidad de que los exosomas funcionen como una nueva forma de comunicación intercelular para establecer y mantener circuitos cerebrales está comenzando a ser explorada. Los exosomas son liberados desde células e interactúan con otras células receptoras para mediar cambios fisiológicos. Todas las células cerebrales liberan exosomas incluyendo las celulas madre neuronales, las neuronas, astrocitos, microglia, oligodendrocitos y las celulas endoteliales. El objetivo de esta revisión es reunir evidencia actualizada sobre las funciones de protección, antiinflamación y regeneración de los exosomas en el ataque cerebrovascular (ACV) isquémico en ratas. Se realizó una búsqueda sistemática de la literatura sensible y específica en base de datos Medline, EMBASE, Web of Science, Scopus, TRIP database, SciELO y LILACS con términos libres y meSH. Los exosomas generados de CSMs pueden ser utilizados para el tratamiento del ACV. Los exosomas de oligodendrocitos también ejercen una variedad de efectos sobre las neuronas receptoras e influencian un amplio espectro de la fisiología neuronal. En conjunto estos resultados sugieren que los exosomas de las CSMs mediados con miR-133b se transfieren a astrocitos y neuronas, las que regulan la expresión génica, beneficiando tanto la remodelación de neuritas, como la recuperación funcional despues de un ACV. Sería importante en el futuro desarrollar métodos para cuantificar y caracterizar los exosomas en el cerebro con isquemia. Esto permitiría correlacionar entre la cantidad de exosomas en el cerebro y la recuperación funcional entregando información sobre sus mecanismos de acción.

PALABRAS CLAVE: Exosomas; Neuroprotección; Isquemia cerebral; Ataque cerebrovascular; Ratas.

\section{INTRODUCCIÓN}

Hasta 1990 los exosomas fueron considerados como el mejor bioproducto de la homeostasis celular. El término exosoma fue inicialmente usado para vesículas de 40 a 1000 nm que son liberadas por una gran variedad de células. Luego, esta nomenclatura fue adoptada para vesículas liberadas durante la diferenciación de reticulocitos como consecuencia de la fusión de un endosoma multivesicular con la membrana plasmática.

Actualmente los exosomas se definen como vesículas liberadas al espacio extracelular a partir del endosoma (Raposo \& Stoorvegel, 2013) y como microvesículas con rango de 40-100 nm (Qin \& Xu, 2014). Los exosomas poseen membrana y otros componentes citosólicos que incluyen proteínas, lípidos, ARN, una composición que difiere dependiendo de su sitio de biogénesis. Muchos mecanismos están involucrados en la formación de vesículas extracelulares, lo cual se refleja en su heterogeneidad, tamaño y composición (Lo Cicero et al., 2015).

La posibilidad de que los exosomas, funcionen como una nueva forma de comunicación intercelular para establecer y mantener circuitos cerebrales está comenzando a ser explorada. Los exosomas son liberados desde células e interactúan con otras células receptoras para mediar cambios fisiológicos (Sharma et al., 2013). Todas las células cerebrales liberan exosomas (Emanueli et al., 2015) incluyendo las células madres neuronales, neuronas, astrocitos, microglia, oligodendrocitos y células endoteliales. Resulta de interés investigar sobre este tema por la prevalencia tanto a nivel mundial como a nivel nacional de enfermedades cerebrovasculares y a que esta área en relación a exosomas y sus aplicaciones ha sido poco investigada en seres humanos.

\footnotetext{
" Doctorado en Ciencias Morfológicas, Facultad de Medicina, Universidad de la Frontera, Temuco, Chile.

*** Escuela de Ciencias de la Salud,Universidad Católica de Temuco, Chile

***: Departamento de Cirugía, Traumatología y Anestesiología, Universidad de la Frontera, Temuco, Chile.

***** Doctorado en Ciencias Médicas, Universidad de la Frontera, Temuco, Chile.

******* Doctorado en Psicología, Salud y Calidad de Vida, Universidad de Girona, Girona, España.
} 
Para comprender el potencial terapéutico de los exosomas en enfermedades neurológicas cerebrales es necesario determinar como ellos atraviesan la barrera hematoencefálica y mejorar los métodos de aislación, carga, caracterización y blanco de diferentes tipos celulares. Los exosomas son producidos por diferentes tipos de células, sin embargo se debe investigar la mejor fuente de células productoras. Las células dendríticas y células mesenquimales son usadas actualmente, pero la inducción de células madres pluripotenciales son una gran promesa ya que tienen una larga vida, pueden ser almacenadas y derivadas desde los fibroblastos de los pacientes, con un bajo riesgo de reacción inmune (Wood et al., 2011).

Los exosomas son liberados por la mayoría de las células bajo condiciones fisiológicas, por otro lado la activación celular o las transformaciones neoplásicas aumentan su liberación (Xin et al., 2013b). En el sistema nervioso, los exosomas participan en la comunicación entre ARNs. Estos exosomas pueden actuar localmente dentro de la terminal presináptica o son transportados hacia el soma de la neurona presináptica (Xin et al., 2013a). Actualmente se piensa que las CSMs (células del estroma mesenquimal) promueven la recuperación neurológica a través de su interacción con células del parénquima cerebral. Las CSMs producen e inducen en las células parenquimatosas efectos biológicos tales como: factores neurotróficos, proteasas y morfógenos; los cuales mejoran el microambiente neurovascular que rodea un area dañada por la isquemia cerebral, así como la remodelación del tejido. Es por esta razón que se hace necesario reunir evidencia actualizada sobre las funciones de protección, antiinflamación y regeneración de los exosomas en el ataque cerebrovascular (ACV) isquémico en ratas.

\section{MATERIAL Y MÉTODO}

Diseño: Revisión bibliográfica, búsqueda sistemática de la literatura (Arias et al., 2012; Manterola et al., 2013; Santander et al., 2013)

Pregunta de búsqueda: ¿Cuál es la función de los exosomas en ratas con enfermedad cerebral isquémica en términos de protección, antiinflamación y regeneración?

Fuentes de búsqueda: PubMed, EMBASE, Web of Science, Scopus, TRIP database, SciELO y LILACS. La búsqueda fue realizada el 31 de Agosto de 2015.

Estrategia de búsqueda: Con términos libres y términos meSH sin límites. Se inicia una búsqueda sensible y luego especifica.
Límites: sin límites. Solo en Scopus se utilizaron límites y estos fueron: "Biochemistry Genetics and Molecular Biology y Neurosciences".

\section{Comandos utilizados en cada base de datos:}

Pubmed: $\quad(((()((()($ exosome) OR microvesicle) $)$ OR microparticle) OR apoptotic body) OR microvesicles) OR "Exosomes"[Mesh]) OR "Endocytosis"[Mesh])) AND (((((Neuron protection) OR neuron Regeneration) OR neuron function protection) $\mathrm{OR}$ neuron function regeneration) $\mathrm{OR}$ neuron anti inflammation $))$ AND $((()(()(()((($ Brain neurons) OR Brain Neuron) OR Brain Presynaptic cell) OR Brain Postsynaptic cell) OR Oligodendrocyte) OR Brain dendritic cells) OR cerebral dendritic cells) OR microRNA) OR brain inflammatory disease) OR cerebral nervous system) OR brain nervous system) OR "Oligodendroglia"[Mesh]) OR "MicroRNAs"[Mesh]) OR "Brain Diseases"[Mesh])) AND (()(()((()((rat) OR genus Rattus) OR Degeneration of cerebral axons) OR Ischaemic Stroke) OR Ischemic Stroke) OR synaptic) OR synaptic function) OR "Rats"[Mesh]) OR "Mice"[Mesh]) OR "BrainIschemia"[Mesh]) OR "Synaptic Transmission"[Mesh]) OR "Hypoxia-Ischemia, Brain"[Mesh]) OR "Cerebral Infarction"[Mesh])) AND ((()((Neuron communication) OR Intercellular comunications) OR exosome release) OR "Cell Communication"[Mesh])) OR "Neurotransmitter Agents"[Mesh])

EMBASE: (Stroke OR brain ischemia OR Ischemia) AND Rats AND Exosomes

Web of Science: Exosomes AND Rats AND Stroke

Scopus: ((Neuron protection OR Neuron antiinflammation OR Neuron regeneration) AND (Stroke OR brain ischemia OR ischemia) AND exosomes AND rats)

\section{TRIP database: Exosomes AND Rats AND Stroke}

\section{SciELO: Exosomes}

\section{LILACS: Exosomes AND Rats AND Stroke.}

Criterios de inclusión: Articulos de investigacíon de exsomas y sus funciones de protección, antiinflamación y regeneracion. Articulos en ratas con enfermedad cerebral isquémica.

Criterios de exclusión: Artículos de investigación en estructuras del sistema nervioso diferentes al cerebro y su vasculatura, como por ejemplo la retina, enfermedades neurodegenerativas como el Parkinson y el Alzheimer o en enfermedades infecciosas, y un estudio realizado en seres humanos (Tabla I). 
Tabla I. Referencias bibliográficas obtenidas según bases de datos consultadas.

\begin{tabular}{lcccc}
\hline Fuente & Artículos obtenidos & Descartado por título y resumen & Duplicados & Artículos atingentes \\
\hline PubMed & 11 & 9 & 0 & 2 \\
EMBASE & 6 & 4 & 0 & 2 \\
Web of Science & 5 & 2 & 2 & 1 \\
Scopus & 57 & 54 & 1 & 1 \\
TRIP database & 1 & 0 & 1 & 0 \\
SciELO & 3 & 1 & 2 & 0 \\
LILACS & 3 & 1 & 2 & 0 \\
\hline
\end{tabular}

Evaluación calidad metodológica de los artículos en extenso: Los 6 estudios fueron sometidos a análisis de calidad metodológica aplicando una puntuación que ha sido previamente validada y publicada (Manterola et al., 2003; Vial et al., 2005; Manterola et al., 2007; Moraga et al., 2013). Está compuesta por 3 ítems: el primero, relacionado con el tipo de diseño del estudio; el segundo, con el tamaño de la población estudiada ajustada según haya o no justificación del tamaño de la muestra y el tercero, relacionado con la metodología empleada en el estudio en cuestión (objetivos, justificación del diseño, criterios de inclusión de la muestra y justificación de ésta). De esta forma, se generó un baremo que es la suma de los ítems 1,2 y 3 , cuya puntuación final puede fluctuar entre 6 y 36, asignándose 6 puntos al estudio de menor calidad metodológica y 36 al de mejor calidad metodológica, con un punto de corte de calidad de 18 puntos (Tabla II).

Tabla II. Escala MINCIR de Calidad Metodológicas para estudios de terapia (Moraga et al.,2014)

\begin{tabular}{|c|c|}
\hline Dominio 1. Diseño del estu dio & Puntaje asigna do \\
\hline Ensayo clínico multicéntrico & 12 \\
\hline Ensayo clínico controlado, con asignación aleatoria y doble enmascarami ento * & 9 \\
\hline Ensayo clínico sin asignación aleatoria, con enmasc arami ento simp le o sin enmascaramiento** & 6 \\
\hline Cohorte concurrente o prospectiva & 4 \\
\hline Cohorte histórica o retrospectiva y estudio de casos y control es & 3 \\
\hline Corte transversal & 3 \\
\hline Series de casos y reportes de casos & 1 \\
\hline Dominio 2. Población estudiada por factor de justificación & Puntaje asigna do \\
\hline $\begin{array}{l}\geq 201 \\
151-200\end{array}$ & $\begin{array}{l}6 \text { ó } 12 \\
5 \text { ó } 10\end{array}$ \\
\hline $101-150$ & 4 ó 8 \\
\hline $61-100$ & 3 ó 6 \\
\hline $31-60$ & 2 ó 4 \\
\hline$<30$ & 1 ó 2 \\
\hline Domin io 3. Metodología empleada & Puntaje asig nado \\
\hline \multicolumn{2}{|l|}{ Item 1.Objetivo } \\
\hline -Se plantean objetivos claros y concretos & 3 \\
\hline -Se plantean objetivos vagos & 2 \\
\hline -No se plantean objetivos & 1 \\
\hline \multicolumn{2}{|l|}{ Item 2. Diseño } \\
\hline -Se menciona y justifica el diseño empleado & 3 \\
\hline -Se menciona el diseño empleado & 2 \\
\hline -No se menciona ni justifica el diseño empleado & 1 \\
\hline \multicolumn{2}{|l|}{ Item 3. Criterios de selección de la muestra } \\
\hline -Se describen criterios de inclusión y de exclusión & 3 \\
\hline -Se describen criterios de inclusión o de exclusión & 2 \\
\hline -No se describen criterios de selección & 1 \\
\hline \multicolumn{2}{|l|}{ Item 4. Tamaño de la muestra } \\
\hline -Justifica la muestra empleada & 3 \\
\hline -No justifica la muestra emp leada & 1 \\
\hline Puntuación final & Puntaje final \\
\hline Dominio $1+($ Dominio $2 \times$ factor de justificación $)+$ Dominio 3 & 6 a 36 \\
\hline
\end{tabular}




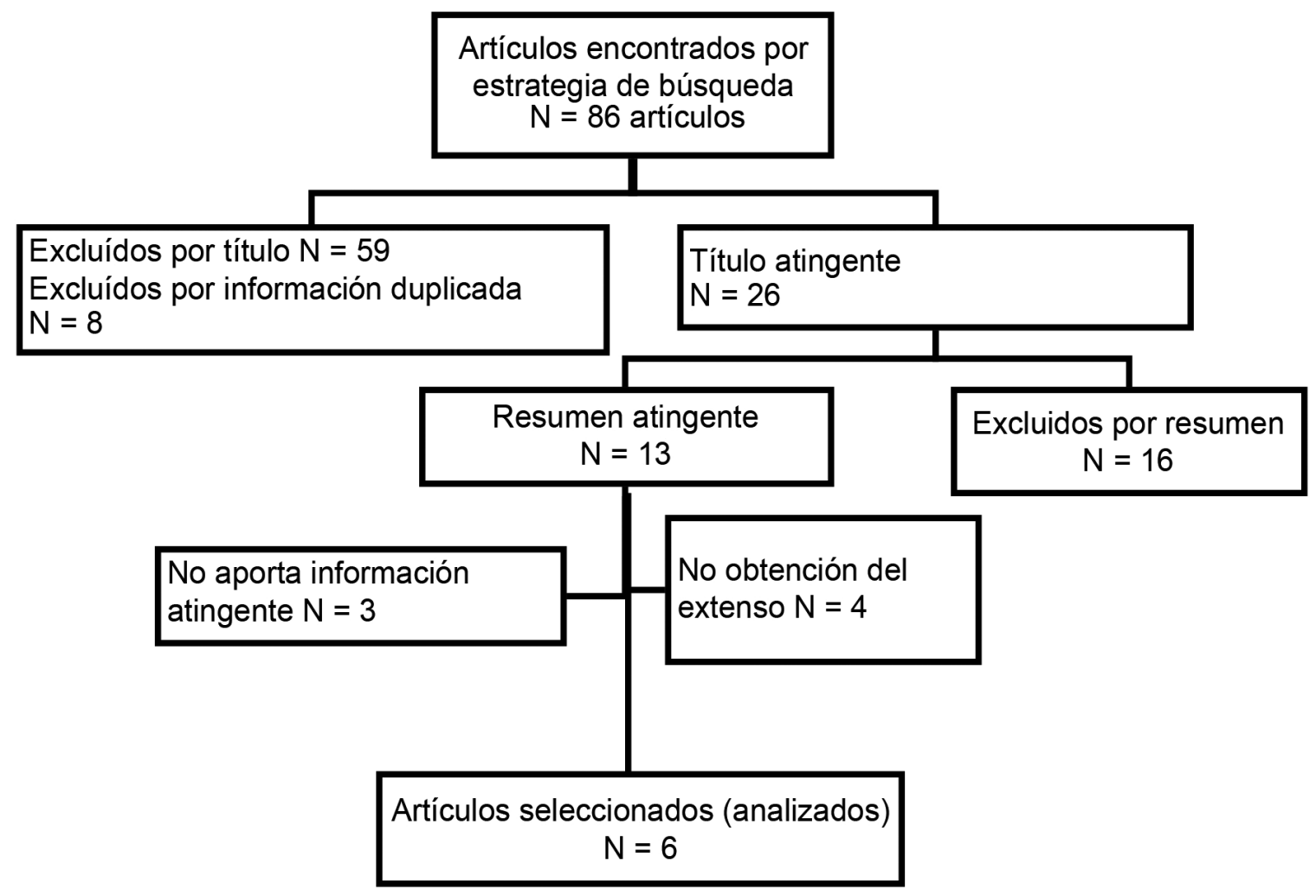

Fig. 1. Flujograma de selección de artículos

\section{RESULTADOS}

Luego de que 13 artículos cumplieron los criterios de inclusión en el resumen, se intentó la obtención de sus extensos. Posterior a la lectura de los extensos, solo seis artículos cumplieron los criterios de inclusión (Fig. 1). En relación a la calidad del reporte de los artículos analizados, cabe mencionar que de estos sólo uno supero los 18 puntos de calidad metodológica, con un promedio de 12,1 \pm 5 puntos y las publicaciones respecto al tema son en promedio del año 2014 (Tabla III).
Los artículos de Xin et al. (2013a), Xin et al. (2013b) y Fröhlich et al. (2015) describieron su diseño del estudio y sus objetivos se plantearon en forma clara y concreta. En relación a los diseños experimentales solo Xin et al. (2013b) explicaron con claridad la población estudiada. En cuanto a la justificación del diseño metodológico se destacaron Drago et al. (2013), Xin et al. (2013a) y Kalani et al. (2015). Ningunos de los artículos señaló los criterios de inclusión y exclusión de la muestra estudiada ni justificó el tamaño de la muestra empleada.

Tabla III. Calidad Metodológica de artículos analizados según escala MINCIR.

\begin{tabular}{lccccccc}
\hline Autor & $\begin{array}{c}\text { Diseño } \\
\text { estudio }\end{array}$ & Población & Objetivo & $\begin{array}{l}\text { Diseño } \\
\text { justificado }\end{array}$ & $\begin{array}{c}\text { Criterios } \\
\text { selección }\end{array}$ & $\begin{array}{l}\text { Tamaño } \\
\text { muestra }\end{array}$ & $\begin{array}{l}\text { Puntaje } \\
\text { final }\end{array}$ \\
\hline Drago et al., 2013 & 1 & $\mathrm{n} / \mathrm{a} *$ & 3 & 3 & 1 & 1 & 9 \\
Fröhlich et al., 2014 & 6 & 2 & 3 & 1 & 1 & 1 & 14 \\
Kalani et al., 2015 & 1 & $\mathrm{n} / \mathrm{a} *$ & 3 & 3 & 1 & 1 & 9 \\
Xin et al., 2013a & 6 & 2 & 2 & 3 & 2 & 1 & 16 \\
Xin et al., 2013b & 6 & 6 & 3 & 1 & 2 & 1 & 19 \\
Xin et al., 2014 & 1 & $\mathrm{n} / \mathrm{a} *$ & 2 & 2 & 1 & $\mathrm{n} / \mathrm{a} *$ & 6 \\
\hline
\end{tabular}

*n/a: no aplica, por tratarse de una revisión bibliográfica. 
Efecto neuroprotector de los exosomas en la isquemia cerebral. En su revisión bibliográfica Kalani et al. refirieron que los exosomas de curcumina encapsulada y los exosomas primarios de curcumina (exosomas liberados por células tratadas con curcumina) es necesario que sean evaluados en relación a su uso como una nueva terapia para el $\mathrm{ACV}$ isquémico. La curcumina es derivada de la raíz de Curcumin longa y debido a sus propiedades medicinales se la ha denominado oro amarillo. La curcumina proporciona un efecto protector vascular en personas con riesgo de ACV. Las propiedades preventivas de ACV pueden ser atribuidas a:1) Neuroprotección vía barrido de radicales libres, inhibiendo la síntesis de óxido nítrico y peroxidación de lípidos. 2) Propiedad antilipidémica por disminución de colesterol y aumento del HDL. 3) Propiedad de antiagregación plaquetaria, inhibiendo la agregación plaquetaria. La habilidad de la curcumina de cruzar la barrera hematoencefalica también favorece su selección sobre otras terapias para ACV. Sin embargo sus desventajas son: pobre absorción, metabolismo rápido y rápida eliminación sistémica.

Fröhlich et al. utilizaron OGD (oxigeno-glucosa deprivación) para simular un modelo in vitro, de isquemia cerebral con muerte celular por necrosis y apoptosis. Los resultados sugirieron que los exosomas de oligodendrocitos liberan enzimas antioxidantes tales como catalasa y SOD1 (superoxido dismutasa 1), lo que confiere la capacidad de resistencia al estrés de las neuronas que reciben a los exosomas.

Efecto antiiflamatorio de los exosomas en la isquemia cerebral. La entrega dirigida de exosomas de curcumina encapsulada al cerebro a través de una vía intranasal, estudiada en ratas, ha sido efectiva para tratar inflamación cerebral. Esta actividad antiinflamatoria puede ser explicada por la supresión de la producción de IL-1, IL-8 y TNF- alfa (Kalani et al.).

Efecto regenerador de los exosomas en la isquemia cerebral. Xin et al. (2013a) administraron exosomas generados por células mesenquimales a ratas con oclusión de la arteria cerebral media y observaron la recuperación funcional, así como los mecanismos de recuperación. 28 días después del $\mathrm{ACV}$ el tamaño de la lesión isquémica fue similar tanto en el grupo control tratado con buffer fosfato salino (PBS) como en el grupo tratado con exosomas. Los exosomas administrados por vía intravenosa, a través de la cola, incrementaron la remodelación de las neuritas en la zona de lesión isquémica. La sustancia blanca en el centro de la lesión fue destruida y los fascículos de axones mielinizados fueron parcialmente dañados y desorganizados después del ACV. La densidad axonal en la zona de la lesión fue incrementada por el tratamiento con exosomas en comparación con el gru- po tratado con PBS. Se observó acumulación de neurofilamentos fosforilados en axones y dendritas después del ACV. Los resultados mostraron que el tratamiento con exosomas aumentó significativamente el área inmunoreactiva SMI-31 en la zona de isquemia comparada con tratamiento con PBS.

La vesícula proteica presináptica sinaptosina es un indicador de plasticidad sináptica y sinaptogénesis. El tratamiento con exosomas aumentó significativamente la densidad axonal y áreas con sinaptosina en la zona de isquemia comparado con el control. Esto sugiere que el tratamiento aumenta la remodelación de neuritas y la plasticidad sináptica en las zonas de isquemia en ratas.

Las células de estroma mesenquimal generaron aumento de exosomas. La neurogénesis y angiogénesis en la zona de isquemia aumentó la proliferación celular en la zona subventricular en el cuerpo estriado y corteza. El tratamiento con exosomas aumento el número de doblecourtin, (marcador de neuroblastos) y el factor von Willebrand (marcador de células endoteliales). Para examinar el efecto de los exosomas sobre los neuroblastos se midió el porcentaje de células en la zona de isquemia en las ratas tratadas con exosomas y en aquellas tratadas con PBS, sugiriendo que los exosomas promueven neurogénesis después del ACV. La angiogénesis involucra proliferación de células endoteliales y de nuevos capilares a partir de vasos ya existentes y se pudo comprobar que los exosomas también promovieron la angiogénesis.

Este estudio mostró que el tratamiento del ACV con exosomas derivados de CSMs, mejoró significativamente los resultados neurológicos y contribuyó la remodelación neurovascular. Los exosomas de las CSMs contienen miRNAs, ARN mensajero y proteínas, las cuales pueden ser transferidas a células receptoras y por lo tanto modificar sus características. Como el miARN tiene un rol primordial en la regulación génica, el miARN encapsulado dentro de los exosomas de las CSMs, tienen un efecto primario en la recuperación de la isquemia. Las condiciones celulares afectan la composición de los exosomas, los exosomas derivados de CSMs expuestos a tejido isquémico contienen miARN aumentado (miARN-133b), el cual facilita la remodelación de neuritas.

Xin et al. (2013b) emplearon las tecnologías knockin y knock down para aumentar o disminuir el nivel de miR133b en CSMs (miR-133b+ CSMs o miR-133b- CSMs) y sus correspondientes exosomas como ganancia o pérdida de la función de miR-133b y luego fueron administradas esas CSMs a ratas sometidas a oclusión de la arteria cerebral media (ACM) para comprobar in vivo si los exosomas 
transfieren miR-133b a las células nerviosas y promueven la remodelación y recuperación funcional. El miR-133b en CSM induce recuperación funcional, esto fue comprobado a través del test de la caída del pie. El aumento en la expresión de miR-133b en CSMs y su liberación de exosomas mejoró la recuperación funcional después del ACV y también demostró remodelación en las neuritas en las zonas de lesión isquémica al día 14 después de la oclusión de la ACM. Etiquetando exosomas con una proteína fluorescente verde se demostró que los exosomas enriquecidos con partículas extracelulares fueron liberados por las CSMs y transferidos a los astrocitos y neuronas adyacentes. La expresión de blancos selectivos para miR-133b, factor de crecimiento de tejido conectivo y el gen homólogo ras miembro de la familia A, se observaron significativamente disminuidos en la zona de isquemia después del tratamiento con miR-133b+CSMs, mientras que su expresión se mantuvo a niveles elevados después del tratamiento con miR-133b-CSMs o con tratamiento placebo con CSMs.

La revisión de Xin et al. (2014) se enfocó en los mecanismos paracrinos de los exosomas de las CSM y la regulación y transferencia de miARNs los cuales mejoran la reparación y la recuperación funcional y las perspectivas sobre el desarrollo del rol de las CSM como mediadoras de la transferencia de exosomas. El ACV induce cambios en el perfildel miARN de las CSMs y en su liberación de exosomas y el miARN participa activamente en los procesos de recuperación después del ACV (Xin et al., 2013a). La liberación de siARN funcional en el cerebro de ratón por inyección sistémica de exosomas, es posible dirigirla a las células nerviosas modificando la membrana proteica exosomal (Xin et al.,).

Drago et al. en su revisión bibliográfica, se refirieron a la más reciente identificación de factores secretados de CSMs y CNP (células madres nerviosas/progenitoras), incluyendo aquellos que son transportados dentro de exosomas y sus potenciales efectos sobre la reparación cerebral. También examinaron los avances en perfiles moleculares que han posibilitado el mapeo de células madres (CMs). Sin embargo como son vesículas con membrana bilipídica, con muchas proteínas ligadas a membrana y diversos contenidos, los exosomas pueden representar un agente terapéutico que tiene el potencial de tratar enfermedades neurológicas. Con este objetivo en mente, es importante realizar una caracterización de los factores neurotróficos y regenerativos presentes en los exosomas secretados tanto por CSMs como por células madres neuroprogenitoras, en un intento de seleccionar e identificar las moléculas responsables del efecto terapéutico de los secretomas de las células madres. Los exosomas tienen efectos beneficiosos similares en terapias regenerativas, la pro- ducción de exosomas de células madres podría ser modificada promoviendo terapias para le reparación de tejido cerebral. Esto se podría conseguir a través de manipulación in vitro de células madres para mejorar la secreción de factores proregenerativos y el desarrollo de nuevas estrategias terapéuticas usando exosomas generados in vitro, enriquecidos con factores de crecimiento, citoquinas, quimioquinas y miRNAs regulatorios que promuevan regeneración del daño tisular.

Fröhlich et al. refirieron que la administración de exosomas de oligodendrocitos influencia la actividad neuronal aumentando el número de potenciales de acción sin alterar la amplitud de su espiga. Los exosomas derivados de microglia actúan sobre la terminal presináptica, en la sinapsis excitatoria, incrementando la liberación de vesículas sinápticas a través de la inducción del metabolismo de esfingolípidos. Un mecanismo similar debiera ser el responsable del incremento de la actividad neuronal a través de los exosomas derivados de oligodendrocitos.

Uno de los efectos de la señalización puede ser la activación o represión de ciertos genes por fosforilación del factor de transcripción cAMP. Adicionalmente los exosomas tienen distintos mARNs y miARNs los cuales pueden presentar efectos sobre la expresión génica neuronal. Los resultados indicaron que los exosomas de oligodendrocitos alteran la expresión génica neuronal y de identifico Ier3, Vgf y Bdnf como genes blanco silenciados en respuesta a la transferencia horizontal desde los oligodendrocitos a las neuronas.

La transferencia de exosomas de oligodendrocitos disminuye la expresión doblecortin neuronal. Los exosomas de oligodendrocitos pueden influenciar la expresión génica neuronal por liberación de miARNs por mediación del gen silenciado. Los precursores de oligodendroglia acumulados en las lesiones del SNC pueden estar involucrados en los procesos de reparación del cerebro a través de estimulación de la regeneración axonal y la secreción de exosomas.

\section{DISCUSION}

Los exosomas generados de CSMs pueden ser utilizados para el tratamiento del ACV (Xin et al., 2013a). Los exosomas de oligodendrocitos ejercen una variedad de efectos sobre las neuronas receptoras e influencian un amplio espectro de la fisiología neuronal (Fröhlich et al.). En conjunto, estos resultados sugieren que los exosomas de las CSMs mediados con miR-133b se transfieren a astrocitos y neuronas, las cuales regulan la expresión génica, beneficiando tanto la remodelación de neuritas como la recuperación funcional después del ACV (Xin et al., 2013a). 
Los exosomas, por su mezcla de factores paracrinos, son ideales para el tratamiento del ACV. En contraste con células exógenas administrada en forma sistémica, los exosomas a causa de su pequeña dimensión pueden atravesar fácilmente la barrera hematoencefálica. La administración exógena de CSMs puede tener muchos efectos adversos tales como modulación o transformación maligna de tumores y pueden obstruir vasos sanguíneos de pequeño calibre. Los exosomas por su pequeño tamaño no causan obstrucción vascular ni se han referido otros efectos adversos relacionados con su uso. Los productos de los exosomas requieren de una amplia caracterización y aislación con el fin de asegurar su función biológica y evitar efectos adversos en el tratamiento del ACV isquémico.

Por ser estudios recientemente desarrollados, es conveniente impulsar y desarrollar nuevos proyectos de investigación que aporten más evidencia dada las proyecciones de la terapia con exosomas. El uso de exosomas en reparación cerebral aún no ha sido bien caracterizada en modelos in vivo. (Drago et al.). Se requiere explicar en forma detallada de qué modo los exosomas participan en los procesos de neuroprotección, antiinflamación y regeneración del tejido cerebral. Si el aspecto epigenético de la curcumina en relación a su potencial neuroprotector para tratar la isquemia cerebral llega a ser aclarado, podrá constituir una nueva terapia para esta enfermedad (Kalani et al.).

Una importante contribución sería poder desarrollar métodos para cuantificar la cantidad de exosomas en el cerebro con isquemia lo que permitiría correlacionar entre los niveles de exosomas en el cerebro y la recuperación funcional proporcionando información sobre sus mecanismos de acción. El desarrollo de tratamientos con células madres locales versus sistémicas que utilizan exosomas, en vez de la célula madre completa, está emergiendo como un exitoso nuevo concepto en medicina regenerativa (Drago et al.).

CARVAllO, P. \& ASTUDILLO, P. Therapeutic effect of exosomes on ischemic stroke in experimental animals. Int. J. Morphol., 34(4):1300-1307, 2016.

SUMMARY: The possibility that exosomes function as a new form of inter cellular communication to establish and maintain brain circuits is beginning to be investigated. Exosomes are released from cells and interact with other receptor cells to mediate physiological changes. All brain cells release exosomes including neural stem cells, neurons, astrocytes, microglia, oligodendrocytes and endothelial cells. The aim of this review is to gather current evidence on the protective, anti-inflammatory and regenerative functions of exosomes in ischemic stroke in rats. A systematic search of sensitive and specific literature was carried out in the following database search engines: Medline, EMBASE, Web of Science, Scopus, TRIP database, SciELO and LILACS with free and MeSH terms data. MSC generated exosomes can be used in the treatment of stroke. Oligodendrocyte exosomes also exert a variety of effects on receptor neurons and influence a wide spectrum of neuronal physiology. Together these results suggest that MSC exosome-mediated transfer of miR-133b to astrocytes and neurons, thus regulating gene expression, benefiting both neurite remodeling, such as functional recovery following a stroke. It would be important in the future to develop methods to quantify and characterize exosomes in brain ischemia. This would allow correlation between the amount of exosomes in the brain and functional recovery providing information relevant to its action mechanisms.

KEY WORDS: Exosomes; Neuroprotection; Brain chemia; Cerebrovascular attack; Rats.

\section{REFERENCIAS BIBLIOGRÁFICAS}

Arias, E.; Astudillo, P.; Manterola, C. \& Grupo MINCIR (Metodología e Investigación en Cirugía). Segunda neoplasia tras el tratamiento del cáncer prostático localizado. Actas Urol. Esp., 36(10):583-9, 2012.

Drago, D.; Cossetti, C.; Iraci, N.; Gaude, E.; Musco, G.; Bachi, A. \& Pluchino, S. The stem cell secretome and its role in brain repair. Biochimie, 95(12):2271-85., 2013.

Emanueli, C.; Shearn, A. I.; Angelini, G. D. \& Sahoo, S. Exosomes and exosomal miRNAs in cardiovascular protection and repair. Vascul. Pharmacol., 71:24-30, 2015.
Fröhlich, D.; Kuo, W. P.; Frühbeis, C.; Sun, J. J.; Zohendner, C. M.; Luhmann, H. J.; Pinto, S.; Toedling, J.; Trotter, J. \& Krämer-Albers, E. M. Multifaceted effects of oligodendroglial exosomes on neurons: impact on neuronal firing rate, signal transduction and gene regulation. Philos. Trans. R. Soc. Lond. B. Biol. Sci., 369(1652):20130510, 2015.

Kalani, A.; Kamat, P. K.; Kalani, K. \& Tyagi, N. Epigenetic impact of curcumin on stroke prevention. Metab. Brain Dis., 30(2):427-35, 2015.

Lo Cicero, A.; Stahl, P. D. \& Raposo, G. Extracellular vesicles shuffling intercellular messages: for good or for bad. Curr. Opin. Cell Biol., 35:69-77, 2015. 
Manterola, D. C.; Pineda, N. V.; Vial, G. M.; Losada, M. H. \& Muñoz, N. S. Revisión sistemática de la literatura. Propuesta metodológica para su realización. Rev. Chil. Cir., 55(2):204-8, 2003.

Manterola, C.; Pineda, V.; Vial, M. \& Astudillo, P. Utilización de analgésicos opiáceos en el proceso diagnóstico y de toma de decisiones sobre pacientes con dolor abdominal agudo no traumático. Revisión sistemática de la literatura. Cir. Esp., 81(2):91-5, 2007.

Manterola, C.; Astudillo, P.; Arias, E.; Claros, N. \& Grupo MINCIR (Metodología e Investigación en Cirugía). Revisiones sistemáticas de la literatura. Qué se debe saber acerca de ellas. Cir. Esp., 91(3):149-55, 2013.

Moraga, C. J.; Burgos, D. M. E.; Manterola, D. C.; Sanhueza, C. A.; Cartes-Velásquez, V. R.; Urrutia, V. S. \& Grupo MINCIR (Metodología e Investigación en Cirugía). Confiabilidad de la escala MINCIR para valorar calidad metodológica de estudios de terapia. Rev. Chil. Cir., 65(3):222-7, 2013.

Moraga, C. J. Manterola, C.; Cartes-Velásquez, V. R.; Burgos, D. M. E.; Aravena, P. \& Urrutia, S. Instrucciones para la utilización de la Escala MINCIR para valorar calidad metodológica de estudios de terapia. Int. J. Morphol.,32:(1)294-8, 2014

Qin, J. \& Xu, Q. Functions and application of exosomes. Acta Pol. Pharm., 71(4):537-43, 2014.

Raposo, G. \& Stoorvegel, W. Extracellular vesicles: exosomes, microvesicles, and friends. J. Cell Biol., 200(4):373-83, 2013.

Santander, A. C.; Astudillo, D. P. \& Manterola, D. C. Procedimiento de Hartmann vs resección y anastomosis primaria en peritonitis diverticular de colon izquierdo por cirugía abierta. Revisión sistemática de la literatura. Rev. Chil. Cir., 65(3):271-8, 2013.

Sharma, P.; Schiapparelli, L. \& Cline, H. T. Exosomes function in cell-cell communication during brain circuit development. Curr. Opin. Neurobiol., 23(6):997-1004, 2013.

Vial, G. M.; Manterola, D. C.; Pineda, N. V. \& Losada, M. H. Coledocolitiasis. Elección de una terapia basada en la evidencia. Revisión sistemática de la literatura. Rev. Chil. Cir., 57(5):404-11, 2005.

Wood, M. J.; O’ Loughlin, A. J. \& Samira, L. Exosomes and the blood-brain barrier: implications for neurological diseases. Ther. Deliv., 2(9):1095-9, 2011.

Xin, H.; Li, Y.; Cui, Y.; Yang, J. J.; Zhang, Z. G. \& Chopp, M. Systemic administration of exosomes released from mesenchymal stromal cells promote functional recovery and neurovascular plasticity after stroke in rats. J. Cereb. Blood Flow Metab., 33(11):1771-5, 2013a.
Xin, H.; Li, Y.; Liu, Z.; Wang, X.; Shang, X., Cui, Y.; Zhang, Z. G. \& Chopp, M. MiR-133b promotes neural plasticity and functional recovery after treatment of stroke with multipotent mesenchymal stromal cells in rats via transfer of exosomeenriched extracellular particles. Stem Cells, 31(12):2737-46, 2013 b.

Xin, H.; Li, Y. \& Chopp, M. Exosomes/miRNAs as mediating cellbased therapy of stroke. Front. Cell. Neurosci., 8:377, 2014.

\section{Dirección de correspondencia: \\ Pamela Carvallo Semler \\ Universidad Catolica de Temuco \\ Manuel Montt 56 \\ Temuco \\ CHILE}

Email:pcarvallosemler@yahoo.es

Recibido : 20-05-2016

Aceptado : 27-08-2016 